\title{
HeartBeat Connections: A Rural Community of Solution for Cardiovascular Health
}

\author{
Gretchen A. Benson, BA, Abbey Sidebottom, MPH, Jeffrey J. VanWormer, PhD, \\ Jackie L. Boucher, MS, Charles Stephens, MD, and Joan Krikava, MD
}

Background: Cardiovascular disease (CVD) continues to be the leading cause of death among Americans. National guidelines emphasize early identification and control of CVD risk factors, but challenges remain in the primary care setting in terms of engaging patients and improving medical therapy adherence. The rapid growth of electronic health records (EHRs) provides a new way to proactively identify populations of high-risk patients and target them with prevention strategies. The HeartBeat Connections (HBC) program was developed as part of a population-based demonstration project aimed at reducing myocardial infarctions.

Methods: HBC uses EHR data to identify residents at high CVD risk in a rural community. Participants receive coaching from a registered dietitian or a registered nurse focused on lifestyle behavior changes and preventive medication initiation/titration.

Discussion: HBC provides patients with access to nonprescribing professionals on a more frequent basis than typical office visits, and it is focused specifically on helping patients improve lifestyle behaviors and medication adherence as they relate to the primary prevention of CVD.

Conclusion: Innovative population health approaches that use EHR data to address common barriers to CVD prevention and engage communities in addressing population health needs are needed to help more patients prevent coronary events. (J Am Board Fam Med 2013;26:299-310.)

Keywords: Patient Care Team, Primary Health Care, Delivery of Health Care, Rural Health

Cardiovascular disease (CVD) continues to be the primary cause of death for American men and women, particularly in rural areas. ${ }^{1,2}$ A national study found that the prevalence of coronary heart disease was 39\% higher among people living in rural areas than among their urban counterparts. These disparities are a function of many social, environmental, and cultural factors but seem to culminate in the underutilization of primary and preventive care. ${ }^{3}$ The prevalence of CVD remains higher for rural residents even after adjusting for CVD risk factors such as poverty, obesity, and tobacco use. ${ }^{4}$

This article was externally peer reviewed.

Submitted 1 September 2012; revised 5 December 2012; accepted 4 January 2013.

From the Department of Education, Minneapolis Heart Institute Foundation, Minneapolis, MN (GAB, JLB); the Center for Healthcare Research \& Innovation, Allina Health, Minneapolis, MN (AS); the Epidemiology Research Center, Marshfield Clinic Research Foundation, Marshfield, WI (JJV); and the New Ulm Medical Center, New Ulm, $\mathrm{MN}(\mathrm{CS}, \mathrm{JK})$.
National guidelines ${ }^{5,6}$ emphasize early identification and control of CVD risk factors as key to CVD prevention, but many challenges exist in the primary care setting in terms of engaging patients and improving adherence to medical therapies. A recent study examining the knowledge and attitudes of primary care providers (PCPs) in the management of CVD risk found that major gaps remain in PCPs' adherence to recommended preventive care guidelines. The competing demands that physicians face during medical encounters present the principal barrier to the provision of specific CVD services to patients. These demands include pa-

Funding: The Heart of New Ulm Project and the HeartBeat Connections program are funded primarily by Allina Health and is a collaboration of the Minneapolis Heart Institute Foundation, the Center for Healthcare and Research Innovation, the New Ulm Medical Center and the community of New Ulm.

Conflict of interest: none declared.

Corresponding author: Gretchen Benson, 920 E. 28th St, Suite 100, Minneapolis, MN 55407 (E-mail: gbenson@ mhif.org). 
tients' acute care needs, requests, and other chronic illnesses as well as a lack of timely access to relevant clinical data and time limitations resulting from a high patient load. ${ }^{7}$ A retrospective review of primary care office visits across the United States found that the median visit length was 16 minutes and covered a median of 6 topics. ${ }^{8}$

Innovative population health approaches ${ }^{9,10}$ that address common barriers to clinical CVD prevention (eg, lack of time and lack of patient follow-up) and engage communities in defining and addressing population health needs are needed. Achieving targets for blood pressure, lipids, and blood glucose may require extensive lifestyle counseling, education, and pharmacological therapy, with ongoing follow-up to foster long-term success. Integrated, multidisciplinary care teams, informed by population health data, may complement traditional clinicbased primary care for CVD. Nonprescribing health care professionals can provide between-care contact in collaboration with the patients' personal physician and make referrals to other community resources.

One emerging approach to achieving targets for blood pressure, lipids, and blood glucose in highrisk patients is to expand the roles of nonprescribing health professionals and integrate them more fully into the primary care setting in conjunction with behavioral change counseling. Within this approach, professionals such as pharmacists, registered dietitians (RDs), and registered nurses (RNs) can follow an approved treatment algorithm to make basic preventive medication changes for patients without direct consultation with a PCP. ${ }^{11-18}$ Published research has focused primarily on using this approach with patients with established disease. The Diabetes Prevention Program, the most successful and arguably well-known of the primary prevention programs, compared outcomes of participants using only a diabetes medication to those of participants who engaged in behavioral strategies, although a combination approach was not studied. ${ }^{19}$

This article describes a program offered in the context of a broader population health initiative that combines both behavioral lifestyle coaching and medication management for a primary prevention population. The HeartBeat Connections (HBC) program is a phone coaching program operated by a rural medical center in conjunction with Hearts Beat Back: The Heart of New Ulm (HONU) Proj- ect. ${ }^{20} \mathrm{HBC}$ aims to improve biometric and behavioral risk factors in individuals identified as being at high risk for CVD. HBC is innovative in its approach to CVD prevention because it uses data from electronic health records (EHRs) to identify eligible patients and uses principles typically employed by disease management programs in a primary prevention population, including a combination of behavioral lifestyle coaching and medication management, while also operating as an extension of clinical care within a local medical facility. In the interest of informing others who are conducting community- or clinic-based primary CVD prevention programs, this article describes how the HBC program was developed and implemented, including the identification of eligible patients via EHR data, as well as the components of the intervention and its evaluation plan.

\section{Background}

In 2009, the HONU Project launched a multiyear initiative to reduce the rate of myocardial infarctions among adult residents of New Ulm, Minnesota, an agricultural region approximately 100 miles southwest of the Minneapolis-St. Paul metropolitan area. The project is a collaborative partnership among Allina Health, the Minneapolis Heart Institute Foundation, the New Ulm Medical Center (NUMC), and the broader community of New Ulm. The HONU Project ${ }^{20}$ involves interventions in the community, health care, worksites, and the nutrition and built environments.

The overarching vision of the HONU Project is to create a sustained culture of health in New Ulm, with programs and initiatives that can be replicated successfully in other rural communities. Within the nutrition environment, for example, the project has partnered with local restaurants to improve menu offerings and is working with convenience stores to increase healthy "grab and go" snack and beverage options. A grant from the U.S. Department of Agriculture is helping the project to train farmers to expand and market their fruit and vegetable offerings through local farmers' markets and community-supported agriculture drop sites. Volunteers are coordinating events enthusiastically, and employers are embracing worksite wellness initiatives. Community leaders are helping to plan wellness programs, and local organizations are promoting heart-healthy lifestyles. The commitment and 
engagement of the entire community drive the success of all the project's activities.

To help inform HONU initiatives, a 36-member steering committee was established at the project's inception. Volunteer members include representatives from a broad cross-section of the community, including physicians at NUMC, local employers, the City of New Ulm, the chamber of commerce, churches, the school district, local colleges, the Brown County Public Health Department, and the general community. The steering committee meets quarterly under the leadership of the HONU Project director. These meetings generally include a brief update on project progress and related current events (eg, funding, outcomes reports, and staff changes), followed by a detailed discussion of near-term intervention priorities and associated challenges. Members often are split into smaller groups to encourage more intimate brainstorming and vetting of possible solutions to such challenges and other community engagement ideas. The meetings end with a group wrap-up session, during which an action plan is informally endorsed or modified.

One of the key reasons the community of New Ulm was selected for the project was because its residents are served primarily by a single health care facility: NUMC. About $90 \%$ of New Ulm residents are NUMC patients and have an EHR. The EHR enables project planners to address various population segments and disease risk levels with the goal of identifying, implementing, and tracking interventions that will positively influence health.

To obtain a robust baseline assessment, a community screening initiative was conducted in 2009 with a goal of creating a "community diagnosis" that would inform the pending interventions. Nearly half of the target population, defined as residents of the 56073 zip code who were 40 to 79 years of age $(\mathrm{n} \approx 7000)$ attended a community screening. ${ }^{21}$ The screenings included both biomedical data (eg, lipids, high-sensitivity C-reactive protein, and blood glucose), which was uploaded automatically into participants' EHRs, and behavioral data (eg, daily consumption of fruits and vegetables, physical activity level, and perceived stress level), which was entered manually into a specially created flow sheet within participants' EHRs.

Data collected from the 2009 screening identified many adults in New Ulm who did not currently have CVD but were at high near-term risk for developing it. On the basis of local EHR data, $~ 2,500$ residents of the 56073 zip code area who are 40 to 79 years of age were considered to be at high risk for CVD, defined as a $\geq 15 \%$ estimated probability of a CVD event in the next decade. This group mainly includes people with metabolic syndrome (ie, those with multiple elevated CVD risk factors).

Because this group of $\sim 2,500$ community residents is an asymptomatic primary care population without diagnosed CVD or a CVD risk equivalent (eg, diabetes), there are many expected gaps in their levels of optimal preventive care. One particularly notable observed gap was that $>50 \%$ were considered clinically eligible for at least one additional preventive medical therapy that they were not getting (eg, aspirin, a statin, or a blood pressure medication). These initial findings indicated a major untapped opportunity to close gaps in optimal preventive CVD care for a large segment of the target population. The HONU Project leadership identified this as a crucial area to address because $\sim 70 \%$ of all myocardial infarctions that occur in a given year in Minnesota are first-time events, ${ }^{22}$ that is, they occur in people who do not have an existing CVD or diabetes diagnosis.

\section{Intervention Description Program Goals}

In August 2010, the HBC program was launched as part of the HONU Project's health care arm. HBC includes free phone coaching to eligible individuals who were identified proactively through the EHR. The program was designed with a core group of PCPs and patients to serve as an extension of clinical primary care as it relates to CVD prevention. HBC focuses specifically on the intense management of CVD risk factors under a supervised protocol and is designed to complement-not replace- usual care. Primary objectives of the HBC program are to:

- increase the proportion of participants taking a daily CVD preventive medication regimen that includes antiplatelet, antidyslipidemia, and antihypertensive medications when indicated;

- increase the proportion of participants with controlled biometric risk factors, including low-density lipoprotein (LDL) cholesterol $<100 \mathrm{mg} / \mathrm{dL}$ and blood pressure $<130 / 85 \mathrm{~mm} \mathrm{Hg}$; and

- increase the proportion of participants with controlled lifestyle risk factors, specifically, those 
who do not smoke, are not obese, and are 100\% adherent to their medication regimens.

\section{Target Population}

The target population for HBC includes all individuals who:

- are 40 to 79 years of age;

- reside in the 56073 zip code;

- are active patients in the Allina EHR system (ie, who are not deceased and have had $\geq 1$ medical encounter with an Allina-associated health care facility in the previous 5 years);

- have high cardiometabolic risk; ie, $\geq 15 \%$ probability of a CHD event in the next 10 years as determined by Framingham ${ }^{23}$ or Reynolds ${ }^{24,25}$ risk scores, meeting the definition of metabolic syndrome, ${ }^{26}$ or both (Table 1 ).

It excludes all individuals who have CVD, diabetes, or chronic kidney disease; reside in an insti- tution; have a major cognitive or language barrier (as determined by program enrollment staff); or have other active end-stage disease (eg, late stage cancer or pulmonary disease).

\section{Cardiometabolic Risk Assessment}

The assessment of CVD risk is based on the best available information contained in the Allina Health EHR system. Only the most recent EHR data from the previous 3 years is used for risk assessment. The quality of the EHR data were enhanced by entering results from the HONU community health screenings for all screening participants. Screening data included all the components of metabolic syndrome and the Framingham and Reynolds risk scores. CVD risk factors taken into account included age, sex, tobacco use, systolic blood pressure, total cholesterol, high-density lipoprotein cholesterol, highsensitivity C-reactive protein, triglycerides, fasting blood glucose, family history of heart disease, medication use, waist circumference, and body mass index (BMI). Data were extracted from the EHR system to

Table 1. Multivariate Risk Metrics and Their Scoring Components

\begin{tabular}{|c|c|c|}
\hline $\begin{array}{l}\text { Measures of High Cardiometabolic } \\
\text { Risk }\end{array}$ & Measurement Components & Description \\
\hline Metabolic syndrome $e^{26}$ & $\begin{array}{l}\text { - Systolic blood pressure } \geq 130 \mathrm{mmHg} \\
\text { or diastolic blood pressure } \geq 85 \\
\mathrm{mmHg} \\
\text { - Waist circumference } \geq 40 \text { inches } \\
\text { (men) or } \geq 35 \text { inches (women); if } \\
\text { waist circumference is unavailable, } \\
\text { BMI } \geq 30 \mathrm{~kg} / \mathrm{m}^{2} \\
\text { - } \mathrm{HDL} \text { cholesterol }<40 \mathrm{mg} / \mathrm{dL} \text { (men) } \\
\text { or }<50 \mathrm{mg} / \mathrm{dL} \text { (women) } \\
\text { - Triglycerides } \geq 150 \mathrm{mg} / \mathrm{dL} \\
\text { - Fasting blood glucose } \geq 100 \mathrm{mg} / \mathrm{dL}\end{array}$ & $\begin{array}{l}\text { This is the first eligibility criteria calculated } \\
\text { for patients. To meet the definition, } \\
\text { patients must have } 3 \text { of the } 5 \text { risk factors. } \\
\text { Although not associated with a specific } \\
\text { risk percentage in a } 10 \text {-year period, } \\
\text { metabolic syndrome significantly } \\
\text { increases the risk of CVD and diabetes. }\end{array}$ \\
\hline Reynolds CHD risk score ${ }^{24,25}$ & $\begin{array}{l}\text { - Age } \\
\text { - Sex } \\
\text { - Tobacco use } \\
\text { - Systolic blood pressure } \\
\text { - Total cholesterol } \\
\text { - HDL cholesterol } \\
\text { - High-sensitivity C-reactive protein } \\
\text { - Family history of heart disease }\end{array}$ & $\begin{array}{l}\text { This score uses the most information, } \\
\text { including C-reactive protein and family } \\
\text { history of heart disease, in addition to } \\
\text { risk factors in the Framingham risk } \\
\text { score. }\end{array}$ \\
\hline Framingham CHD risk score $\mathrm{e}^{23}$ & $\begin{array}{l}\text { - Age } \\
\text { - Sex } \\
\text { - Tobacco use } \\
\text { - Systolic blood pressure } \\
\text { - Total cholesterol } \\
\text { - HDL cholesterol }\end{array}$ & $\begin{array}{l}\text { This score is assessed if there is no } \\
\text { metabolic syndrome and incomplete } \\
\text { information to assess the Reynolds risk } \\
\text { score. }\end{array}$ \\
\hline
\end{tabular}

BMI, body mass index; CHD, coronary heart disease; CVD, cardiovascular disease; HDL, high-density lipoprotein. 
Figure 1. Flow chart for determining eligibility. BMI, body mass index; CHD, coronary heart disease; EHR, electronic health records; GFR, glomeruler filtration rate; HDL, high-density lipoprotein.

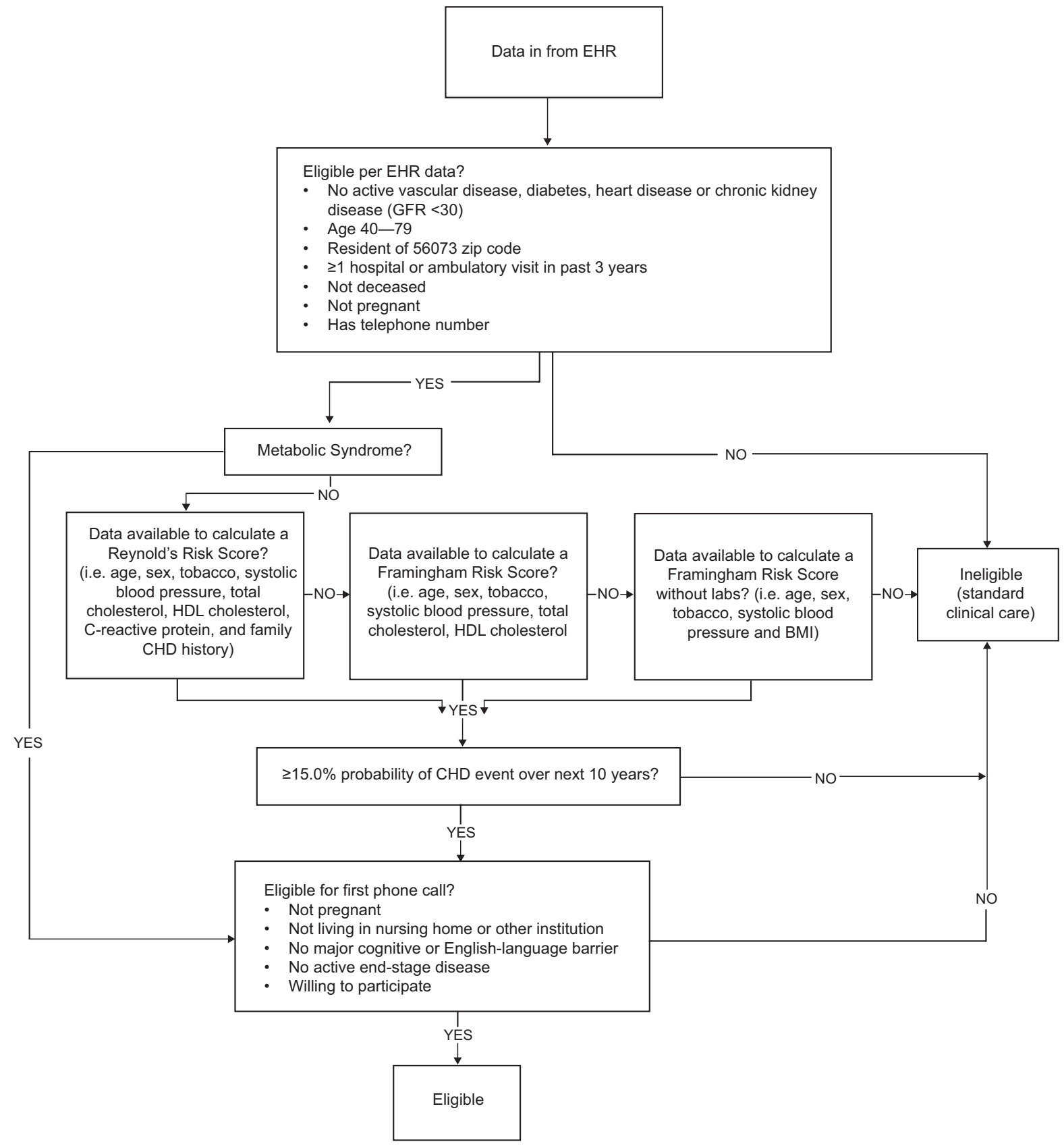

identify individuals who potentially were eligible for the program. CVD risk factors were extracted, taking each person's most recent value for each measure; thus, not all measures were available from the same date.

An analysis of the first 1005 patients who were identified as eligible indicates that the majority had metabolic syndrome (91\%), whereas $\sim 9 \%$ did not have metabolic syndrome but did have a high risk score from either the Framingham or the Reynolds assessment. The process for identifying eligible patients looked first to see if they met the metabolic syndrome criterion; if so, additional eligibility criteria were not checked. Thus, many of those with metabolic syndrome may also have met the other criteria. Data were analyzed to exclude people meeting exclusion criteria and to calculate the measures of high risk (Figure 1). 
Figure 2. HeartBeat Connections program materials, including a booklet about risk reduction, a community resource guide, and a heart-healthy cooking booklet.

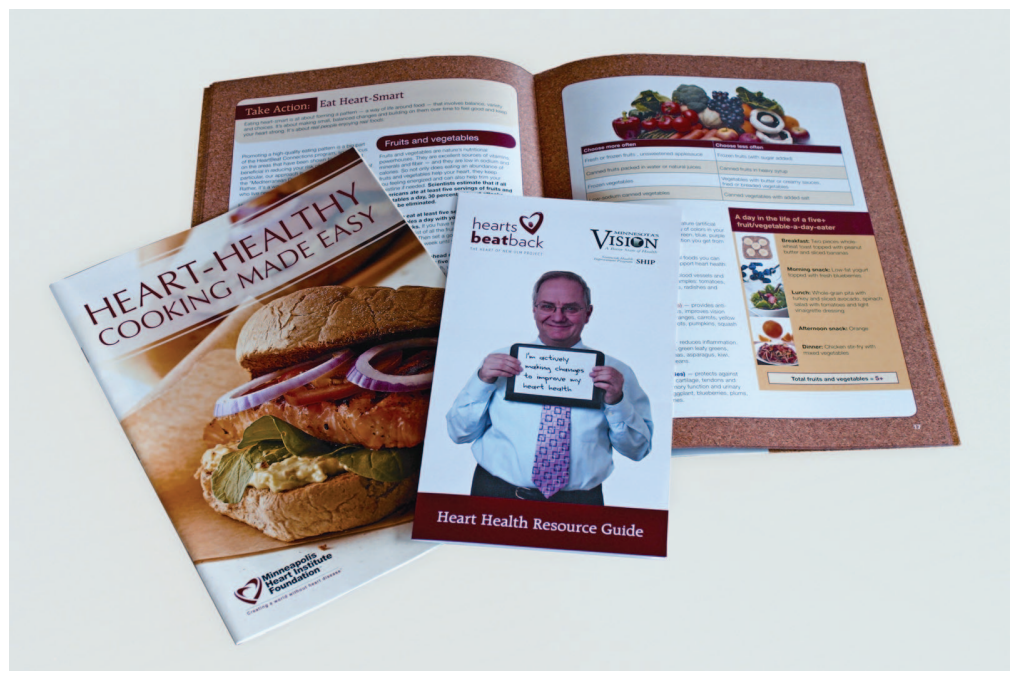

\section{Recruitment and Enrollment}

The list of patients who met the eligibility criteria initially was loaded into a database designed specifically for the HBC program to parallel the EHR in terms of tracking outreach and enrollment activities. Because of the small number of health coaches available, recruitment was done on a rolling basis in batches of approximately 100 to 150 each month. Recruitment goals for year 1 included outreach to the first 1500 high-risk patients identified, followed in year 2 by completion of outreach to the remaining 1000 patients identified.

Recruitment efforts included an invitation letter signed by the project's medical director and a phone number for potential participants to call to enroll or find out more about the program, as well as an "invitation packet" that included a booklet explaining the risk status used to identify eligible patients and tips about lifestyle changes to reduce risk. Patients also received a "Cooking Healthy for Your Heart" booklet (Figure 2) with nutrition information and recipes and a community resources flyer with local resources for targeted risk factors such as weight management, nutrition, physical activity, and smoking. Health coaches made up to 4 attempts to reach eligible patients by phone. After the final attempt, eligible patients were sent a letter giving a final message about the program and letting them know they could call at any time to enroll. Appointments for those who opted in were scheduled in the EHR. Health coaches explained to participants that they would call on a certain date and at a certain time and that phone coaching appointments should be thought of as similar to clinic visits. Participants were encouraged to call into the clinic in advance if they needed to reschedule.

\section{Intervention and Treatment}

$\mathrm{HBC}$ was created as an adjunct to usual care and provides ongoing support, education, and medication guidance between usual clinic visits. HBC phone coaching staff includes 2 RDs (0.5 and 0.25 full-time equivalent) and an $\mathrm{RN}$ (0.8 full-time equivalent) with clinical oversight from a NUMC nurse practitioner who all collaborate with PCPs.

Participants talk with a health coach at 1 - to 2-month intervals, depending on their personal medication therapy regimen and preferences. Phone calls typically last 20 to 30 minutes. These calls are intended to supplement the participants' relationships with their PCP and offer additional support between office visits. Coaches tailor the approach to the individual and may draw on key behavioral theories (eg, motivational interviewing and intrinsic coaching principles). Coaches work with participants to set clinical and behavioral goals between office visits.

Lifestyle behavior change is a primary focus of the phone coaching appointments because PCPs have limited time to address these factors during a typical office visit. Health coaches strongly emphasize nutrition and physical activity according to CVD prevention recommendations. ${ }^{5,27,28}$ Topics 
covered include increasing the number of fruits and vegetables eaten each day, incorporating healthy fats, incorporating fish weekly, reducing sodium, including whole grains, limiting added sugars, and reducing portions and calories. Stress management, tobacco cessation, and weight management also are covered in the booklet that accompanies the coaching visits.

A unique aspect of this program is its use of a medication protocol created with and approved by NUMC physicians that allows the health coaches to initiate and titrate some preventive medical therapies (eg, blood pressure and lipid medications) following an approved treatment decision algorithm. Participants with a recently elevated blood pressure or LDL cholesterol level are asked to take 6 months to try to reduce these values through lifestyle modifications alone. After that time, the coaches may recommend pharmacotherapy if the program goal is unmet. The health coaches review with patients the purpose of these therapies and stress the importance of medication adherence. Further details of the therapy goals are outlined below.

\section{Aspirin}

- Male participants $\geq 45$ years old and women $\geq 55$ years old will be started on aspirin $(81 \mathrm{mg}$ /day $)$ unless contraindications are present.

- Coaches will monitor for side effects (eg, epistaxis, bloody nose, bleeding gums, unusual bleeding/bruising, and gastrointestinal side effects) during follow-up calls; if side effects or intolerance are noted after starting aspirin, a HONU Project nurse practitioner or PCP will be notified.

\section{Statin Therapy (Program Goal for LDL Cholesterol, $<100$ $m g / d L)$}

- A statin (primarily simvastatin) will be recommended if LDL cholesterol is $>100 \mathrm{mg} / \mathrm{dL}$ (based on the most recent measurement within the previous 12 months) unless contraindications are present. For patients with an LDL cholesterol level between 100 and $130 \mathrm{mg} / \mathrm{dL}$, lifestyle changes will be attempted for 6 months before medication is initiated. For patients who enter the program taking a statin other than simvasta- tin, health coaches can titrate the statin according to a preapproved statin equivalency chart.

Angiotensin-Converting Enzyme Inbibitor (Program Goal for Blood Pressure, $<130 / 85 \mathrm{~mm} \mathrm{Hg}$ )

- Lisinopril will be initiated if blood pressure is $>130 / 85 \mathrm{~mm} \mathrm{Hg}$ on at least 3 separate occasions within the previous 12 months, as recorded in the EHR. Participants who enter the program already taking 1 or 2 hypertension medications other than an angiotensin-converting enzyme inhibitor and are still not at the program blood pressure goal may be started on an angiotensin-converting enzyme inhibitor. Other hypertension medications will not be titrated.

- All encounters are documented in the EHR for seamless coordination of care with PCPs. A chart note and a brief lifestyle assessment questionnaire are completed during each phone encounter. Because the EHR design focuses on acute care documentation, a special flowchart was created within the EHR to capture selfreported data related to lifestyle prevention measures such as systematic measures of daily aspirin use, adherence to medications for blood pressure and cholesterol, fruit and vegetable consumption, perceived stress level, physical activity, and smoking status (Table 2). Further communication with providers via a staff message (ie, E-mail sent through the EHR system) may occur when a medication is started; a medication is discussed but the participant declines to use it; a conflicting LDL cholesterol goal is noted in the chart (ie, $>100 \mathrm{mg} / \mathrm{dL}$ ); or a laboratory test is ordered that may need direct follow-up related to diagnosis (eg, glycosylated hemoglobin).

- Participants may remain in the program for as long as needed to make behavior changes. A change is program status is a mutual decision between participant and health coach. Participants are considered to have "completed" the program if they have had three or more coaching visits, meet program goals for blood pressure and LDL cholesterol, and meet at least two of the following criteria: non-smoker, BMI $<30 \mathrm{~kg} / \mathrm{m}^{2}$, and $100 \%$ medication adherence. Participants who choose to disengage from the 


\begin{tabular}{|c|c|c|c|}
\hline Measure & $\begin{array}{l}\text { Reported from Last } \\
\text { Available in EHR }\end{array}$ & $\begin{array}{l}\text { Reported from HBC Flow Sheet } \\
\text { (Self-Reported Data From Each } \\
\text { Coaching Call) }\end{array}$ & Program Goal \\
\hline $\mathrm{BMI}\left(\mathrm{kg} / \mathrm{m}^{2}\right)$ & $\mathrm{X}$ & & $<30$ \\
\hline Blood pressure $(\mathrm{mmHg})$ & $\mathrm{X}$ & & $<130 / 85$ \\
\hline Total cholesterol (mg/dL) & $\mathrm{X}$ & & $<200$ \\
\hline LDL cholesterol (mg/dL) & $\mathrm{X}$ & & $\leq 100$ \\
\hline HDL cholesterol (mg/dL) & $\mathrm{X}$ & & $\geq 40$ For men, $\geq 50$ for women \\
\hline Triglycerides (mg/dL) & $\mathrm{X}$ & & $<150$ \\
\hline Frequency of aspirin use & & $\mathrm{X}$ & $\begin{array}{l}100 \% \text { Adherent if } \\
\text { recommended }\end{array}$ \\
\hline $\begin{array}{l}\text { Frequency of blood pressure } \\
\text { medication use }\end{array}$ & & $\mathrm{X}$ & $\begin{array}{l}100 \% \text { Adherent if } \\
\text { recommended }\end{array}$ \\
\hline Frequency of statin use & & $\mathrm{X}$ & $\begin{array}{l}100 \% \text { Adherent if } \\
\text { recommended }\end{array}$ \\
\hline $\begin{array}{l}\text { Fruit and vegetable servings eaten } \\
\text { each day (n) }\end{array}$ & & $\mathrm{X}$ & $\geq 5$ \\
\hline Physical activity/week (min) & & $\mathrm{X}$ & $\geq 150$ \\
\hline Tobacco status & $\mathrm{X}$ & & Never or quit \\
\hline Overall stress on a scale from $1-10^{*}$ & & $\mathrm{X}$ & $1-3$ \\
\hline
\end{tabular}

${ }^{*} 1=$ Not stressed at all, $10=$ very stressed.

BMI, body mass index; EHR, electronic health record; HBC, HeartBeat Connections program; HDL, high-density lipoprotein; LDL, low-density lipoprotein.

program without meeting the aforementioned criteria are considered to have opted out.

\section{Evaluation Design \\ Process Evaluation}

Because HBC is a new program, process evaluation will focus on the success of enrolling, engaging, and retaining participants. Evaluation of recruitment will help to identify whether there is a need to adjust communication strategies among HBC program staff or the broader PCP group. Process evaluation also will focus on measuring the staff-to-patient ratio as well as the success of coaches (and disciplines) in engaging and retaining patients. Process questions to be examined include, What proportion of eligible patients choose to enroll? How many recruitment phone calls are needed to successfully reach and enroll eligible patients? How do recruitment results differ by phone coach (both individually and by discipline)? How often do phone coaching appointments occur once patients are enrolled?

Two sources of data will be used to answer these questions: patients' EHRs and the previously described database that was developed to track recruitment efforts and program participation. The original goal was to conduct all program documentation in the EHR system because the program is an extension of clinical practice and all coaches are employed by the NUMC. However, the current EHR system is better suited to manage acute care and did not have the capabilities to track outreach attempts systematically (ie, queuing patients in a timely fashion for consecutive outreach attempts, assigning eligible patients to coaches for recruitment or case management, and providing an ondemand reporting structure for each of these items to make timely programmatic adjustments as needed). Thus, the database was designed to provide an outreach list for coaches to follow during assigned recruitment time.

For each coaching call, the database tracks the date, time, and outcome (eg, enrolled, opted out, met exclusion criteria, left message, or unable to leave message). If unavailable, the database queues the next outreach call to occur in 7 days.

Once a patient is successfully reached and makes a decision about participation, or after all 4 recruitment phone calls have been completed without reaching the patient, health coaches indicate the final outcome in both the database and the EHR. Documentation in the EHR uses an order, created specifically for the HBC program, indicating the patient's program participation status: "opt-in" for those who choose to participate and "opt-out" for 
those who did not enroll, with subcategories indicating the reason for nonenrollment (eg, refusal or unreachable).

Analysis to evaluate process outcomes is performed on an ongoing basis, with reports built into the database to examine the recruitment status for all potential enrollees, both as a whole and by coach, to monitor differential recruitment outcomes and identify process improvement opportunities. Recruitment reports also document the percentage of patients enrolling after each number of recruitment phone calls (ie, 1, 2, 3, or 4) to determine whether the program is still successfully gaining enrollees at the fourth call or whether fewer calls are adequate or more calls might be necessary. Recruitment reports also document the proportion of patients who are never reached directly or never respond to messages to determine whether additional recruitment methods are needed.

Additional analyses will be performed using EHR orders to select potentially eligible patients for whom recruitment efforts were completed. A data extract will be completed selecting patients with any HBC order (opt in or opt out) and selecting additional information about those patients, such as their risk factors, age, and sex, to allow comparison between those who enroll to those who do not enroll as well as between the 2 categories of opt-outs (refusal and unreachable).

Database reports also have been built to determine the number of completed appointments among enrollees and by coach. To examine retention, a report captures information about patients who enroll and then opt out, documenting reasons for leaving the program. Further analyses can be performed to determine the patients' duration of enrollment in the program and number of completed appointments. Another report was designed to track the individual patient panel of each NUMC medical provider and the corresponding program status of those patients. These reports are generated bimonthly to inform providers about their patients and about the general performance of HBC.

\section{Outcome Evaluation}

The essential outcome question is: Among HBC eligible individuals, do program enrollees experience greater improvements in CVD risk factors (eg, LDL cholesterol, blood pressure, BMI, and smoking status) relative to those who did not en- roll? Additional outcome evaluation questions include, Among those enrolled, are larger changes in biometric or behavioral risk factors experienced with longer program enrollment or greater number of coaching contacts? Are comparable outcomes observed between coaches with different professional training (eg, dietitians vs nurses)?

For outcome measures that are standard components of the EHR, changes over time will be compared for HBC enrollees and eligible nonenrollees as classified by the HBC-specific order in the EHR system. For outcome measures that are not available as standard components of the EHR but are collected as part of the HBC program, evaluation analysis will examine changes within HBC program enrollees only (Table 2).

To answer outcome evaluation questions, all individuals with an HBC program order (indicating opt-in or opt-out status) will be extracted from the EHR along with baseline CVD risk factor values used to determine program eligibility. The most current values of those same CVD risk factors after enrollment will be collected to assess changes. This sort of real-world program evaluation is vulnerable to selection biases in that program enrollees may differ from those who did not enroll in meaningful ways that can explain observed differences in outcomes. However, efforts will be made to adjust statistically for baseline differences between enrollees and nonenrollees as one way to account for this. For comparisons between enrolled and eligible nonenrolled patients, the analysis will examine mean changes in biometric risk factors and the percentage of patients meeting recommended levels for these risk factors at baseline and after program participation or a similar amount of time for nonparticipants. For comparisons within the group of enrollees, mean values and the percentage meeting program goal levels will be compared from baseline to the most current values, with stratification by number of coaching contacts.

\section{Program Satisfaction}

Physician surveys occur at HONU-sponsored continuing medical education events that are offered biannually to providers. The events, which feature national speakers in the field of preventive cardiology, are held to keep physicians informed about state-of-the-art prevention and to share data and current HONU Project offerings. Physicians have indicated that phone coaching is a new concept for 
them, so future seminars will focus on the clinical utility of phone coaching.

\section{Discussion}

As one component of the broader HONU Project, HBC was created to support PCPs in finding and treating high-risk primary prevention patients within the region. The HBC program provides access to frequent between-visit telephone coaching from RDs and RNs who are trained in CVD risk factor modification related to lifestyle and medication adherence. The program also links participants to other resources within the community (eg, weight management classes, fitness facilities, and farmers' markets), thereby integrating medicine and public health per the recommendations from the Folsom Group.

Because $\mathrm{HBC}$ is an enhancement of clinical care, one of the most important programmatic accomplishments to date has involved establishing a strong level of trust with local PCPs, collaborating with them from conceptual design to ongoing discussions of quality assurance and continued evaluation of the program. Perhaps the best example of this so far has been the collaborative creation of a formal medication protocol. These discussions started by reviewing current literature related to nonprescribing health professionals serving in this role to support and inform facility leaders. Findings were presented to a local leadership group, and consensus was gained to create a clinical policy. An oversight committee also was formed to refine the policy as needed, with members including the hospital president and medical director, a consulting cardiologist, several physicians, a nurse practitioner, a pharmacist, a nurse manager, and the program managers. Monthly planning meetings were held to gather input and construct the protocol. The final step of this process was review and approval of the policy by NUMC's governing body, the Medical Operations Executive Committee, which also reviews and renews its approval annually.

In addition to the support gained from the clinical sector, efforts were made to garner community support for HBC. A focus group was conducted before the program's launch that specifically targeted community residents who would qualify for the program. A group of 9 residents (4 men and 5 women) provided feedback about the general pro- gram concepts and gave specific insights into program design and user flow. Some early privacy concerns were noted regarding using EHR data as an identifier, but the group indicated that such concerns could be largely assuaged by emphasizing the "extended PCP" nature of the program, whereby all staff operated from NUMC and were held to the same privacy standards. The group was overwhelmingly receptive to the telephone outreach process but wanted to be sure their PCPs were kept informed and involved. Similar concerns were raised within the HONU Project community steering committee, and, based on the collective feedback from these 2 community groups, strong consensus to move the program forward eventually was reached.

The technology of the EHR system made the $\mathrm{HBC}$ program, which integrates primary care and the broader community, possible. Because HBC targets a primary prevention population, there is not a single diagnosis or event that easily flags eligibility. Rather, people are deemed eligible based on a complex algorithm that simultaneously considers multiple CVD risk factors measured during both usual office visits and community health screenings conducted periodically. The EHR system also permits more robust evaluations, enabling the efficient comparison of those who participated and eligible nonparticipants.

There are limitations to this approach, however, because it relies on naturalistic office visits during which lipids, blood pressure, and other CVD risk factors are measured. Thus, there is potential for missing data because of infrequent visits to the clinic or measurements not being performed when patients do visit. Our examination of lifestyle behavior factors, which are well-known risk factors for CVD and the largest influencers of premature mortality, ${ }^{29}$ is limited to those participating in the program because standardized measures for these risk factors (eg, physical activity, stress, and fruit and vegetable consumption) do not exist in the EHR system for all patients. These measures are collected during each telephone call with patients and tracked in a flow sheet (a standardized questionnaire built into the EHR system with preloaded response items listed in a drop-down format) that was created specifically for the program. Self-reported data obtained from these calls populate the health coach's chart notes, so a comprehensive overview of each health coach call is available 
within the EHR for review by patients' PCPs, similar to an in-person office visit. Another EHR limitation is the inability to conduct certain logistic functions related to tracking recruitment and appointment calls. As a result, health coaches also track outreach calls and appointment calls in a parallel but separate database.

The model used by HBC to recruit eligible patients is similar to that of other disease management programs, but it remains a major challenge in a primary prevention population to explain the concept of "high risk" to asymptomatic patients. This scenario is different from outreach efforts for individuals with known disease or who have experienced a recent disease event and as a result may be highly motivated to change. Furthermore, it is unknown how receptive individuals in the target population will be to starting a preventive medical therapy on the recommendation of someone other than their PCP. One advantage of this program is that the broader HONU Project is well known among community residents. Therefore, explaining that $\mathrm{HBC}$ is part of a broader and more familiar community initiative may aid enrollment goals.

Future research considerations for the program will be to generate sample size estimates for trial studies with similar interventions being proposed in collaboration with other health care systems. Given the single health care services provider in New Ulm, it will be important for future studies to test the degree to which HBC provides a model of panel management that other communities can replicate. Studies of the cost effectiveness of the program also are being proposed. If HBC proves successful in helping patients reduce their CVD risk, it may be worth exploring creative reimbursement models to sustain the program over the long term as part of standard medical services.

\section{Conclusion}

PCPs face significant time constraints and other barriers to delivering preventive CVD care, as suggested by national guidelines. Innovative population health approaches that incorporate EHR and community data and engage communities in defining and addressing health needs are necessary to help more patients prevent disease. This is one of the recommendations of the revised Folsom Report. ${ }^{9}$ Identification of patients at high-risk for CVD, coupled with the development of collabora- tive approaches that engage other members of the health care team in patient care, can help to ensure that these patients receive appropriate therapeutic regimens and have needed access to ongoing coaching and community resources to aid in sustaining lifestyle changes. The HBC program could potentially serve as a model of how to support PCPs and patients in achieving CVD prevention targets and enhance the broader relationship of health care systems with the communities they serve. Evaluation of this program is needed to provide more definitive conclusions regarding its effect.

The authors gratefully acknowledge the HeartBeat Connections program staff; Julie Long, CNP; Theresa Bunkers-Lawson, RN, CDE; Joy Hayes, RD, CDE; and Kate Callahan Schmitz, RD; New Ulm Medical Center providers; as well as all of the community residents who participate in the program. They also gratefully acknowledge Kevin Graham, MD, whose vision contributed to the conceptualization and development of the Heart of New Ulm Project.

\section{References}

1. Barnett E, Halverson J. Disparities in premature coronary heart disease mortality by region and urbanicity among black and white adults ages 35-64, 1985-1995. Public Health Rep 2000;115:52-64.

2. Quarells RC, Liu J, Davis SK. Social determinants of cardiovascular disease risk factor presence among rural and urban Black and White men. J Mens Health 2012;9:120-6.

3. Colleran KM, Richards A, Shafer K. Disparities in cardiovascular disease risk and treatment: demographic comparison. J Investig Med 2007;55:41522.

4. O'Connor A, Wellenius G. Rural-urban disparities in the prevalence of diabetes and coronary heart disease. Public Health 2012;126:813-20.

5. Expert Panel on Detection, Evaluation, and Treatment of High Blood Cholesterol in Adults. Executive summary of the third report of the National Cholesterol Education Program (NCEP) Expert Panel on Detection, Evaluation, and Treatment of High Blood Cholesterol in Adults (Adult Treatment Panel III). JAMA 2001;285:2486-97.

6. Chobanian AV, Bakris GL, Black HR, et al. Seventh report of the Joint National Committee on Prevention, Detection, Evaluation, and Treatment of High Blood Pressure. Hypertension 2003;42:1206-52.

7. Doroodchi H, Abdolrasulnia M, Foster JA, et al. Knowledge and attitudes of primary care physicians in the management of patients at risk for cardiovascular events. BMC Fam Pract 2008;9:42.

8. Tai-Seale M, McGuire TG, Zhang W. Time allo- 
cation in primary care office visits. Health Serv Res 2007;42:1871-94.

9. Folsom Group. Communities of solution: the Folsom Report revisited. Ann Fam Med 2012;10:250-60.

10. Institute of Medicine. Primary care and public health: exploring integration to improve population health. Washington, DC: National Academies Press; 2012.

11. Andersen UO, Simper AM, Ibsen H, Svendsen TL. Treating the hypertensive patient in a nurse-led hypertension clinic. Blood Press 2010;19:182-7.

12. Davidson MB. How our current medical care system fails people with diabetes: lack of timely, appropriate clinical decisions. Diabetes Care 2009; 32:370-2.

13. New JP, Mason JM, Freemantle N, et al. Specialist nurse-led intervention to treat and control hypertension and hyperlipidemia in diabetes (SPLINT): a randomized controlled trial. Diabetes Care 2003;26: 2250-5.

14. Robinson JG, Conroy C, Wickemeyer WJ. A novel telephone-based system for management of secondary prevention to a low-density lipoprotein cholesterol $<$ or $=100 \mathrm{mg} / \mathrm{dl}$. Am J Cardiol 2000;85: 305-8.

15. Rudd P, Miller NH, Kaufman J, Kraemer HC, Bandura A, Greenwald G, Debusk RF. Nurse management for hypertension. A systems approach. Am J Hypertens 2004;17:921-7.

16. Senior PA, MacNair L, Jindal K. Delivery of multifactorial interventions by nurse and dietitian teams in a community setting to prevent diabetic complications: a quality-improvement report. Am J Kidney Dis 2008;51:425-34.

17. Tobe SW, Pylypchuk G, Wentworth J, et al. Effect of nurse-directed hypertension treatment among First Nations people with existing hypertension and diabetes mellitus: the Diabetes Risk Evaluation and Microalbuminuria (DREAM 3) randomized controlled trial. CMAJ 2006;174:1267-71.

18. Worth JM, Davies RR, Durrington PN. A dietitianled lipid clinic is effective. Pract Diabetes Int 2006; 23:221-6.

19. Knowler WC, Barrett-Connor E, Fowler SE, et al. Reduction in the incidence of type 2 diabetes with lifestyle intervention or metformin. New Engl J Med 2002;346:393-403.
20. Boucher JL, Pereira RF, Graham KJ, Pettingill RR, Toscano JV, Henry TD. The heart of new ulm: a vision for the future. J Cardiovasc Transl Res 2008; 1:310-6.

21. VanWormer JJ, Johnson PJ, Pereira RF, et al. The Heart of New Ulm Project: using community-based cardiometabolic risk factor screenings in a rural population health improvement initiative. Popul Health Manage 2012;15:135-43.

22. McGovern PG, Jacobs DR Jr, Shahar E, et al. Trends in acute coronary heart disease mortality, morbidity, and medical care from 1985 through 1997: the Minnesota heart survey. Circulation 2001; 104:19-24.

23. Wilson PW, D'Agostino RB, Levy D, Belanger AM, Silbershatz H, Kannel WB. Prediction of coronary heart disease using risk factor categories. Circulation 1998;97:1837-47.

24. Ridker PM, Buring JE, Rifai N, Cook NR. Development and validation of improved algorithms for the assessment of global cardiovascular risk in women: the Reynolds Risk Score. JAMA 2007;297:611-9.

25. Ridker PM, Paynter NP, Rifai N, Gaziano JM, Cook NR. C-reactive protein and parental history improve global cardiovascular risk prediction: the Reynolds Risk Score for men. Circulation 2008;118:2243-51, 4p following 2251.

26. Grundy SM, Cleeman JI, Daniels SR, et al. Diagnosis and management of the metabolic syndrome: an American Heart Association/National Heart, Lung, and Blood Institute Scientific Statement. Circulation 2005;112:2735-52.

27. Lichtenstein AH, Appel LJ, Brands M, et al. Diet and lifestyle recommendations revision 2006: a scientific statement from the American Heart Association Nutrition Committee. Circulation 2006;114: 82-96.

28. Lloyd-Jones DM, Hong Y, Labarthe D, et al. Defining and setting national goals for cardiovascular health promotion and disease reduction: the American Heart Association's strategic Impact Goal through 2020 and beyond. Circulation 2010;121: 586-613.

29. Schroeder SA. Shattuck Lecture. We can do betterimproving the health of the American people. New Engl J Med 2007;357:1221-8. 